\title{
On Existence in Set Theory, Part II: Relative Productivity
}

\author{
Rodrigo A. Freire
}

\begin{abstract}
The present paper continues the investigation initiated in an earlier work. After a short introduction, the notion of relative productivity is defined and a technical apparatus is developed in order to evaluate the classification of the axioms previously obtained. Some results on the semilattice of simple relative degrees are proved at the end of Section 2. Section 3 adds some concluding remarks.
\end{abstract}

\section{Introduction}

The analysis of existence assertions in Part I (see [1]) is founded on the notion of productivity: an existence assertion is either productive or admits unconditional degree 0 . The productivity of a (valid) sentence is, roughly, its power of producing sets. This existential power can be measured by the closure property that a domain should have in order to fulfill the existence requirement of the sentence. The plausibility of this identification rests on the intuition that if a valid sentence is not valid in all domains with a specific closure property, then that failure is caused by an insufficiency of the closure property. This identification is interesting because there is a natural hierarchy of closure properties for domains, gradually increasing from no closure property, closure under taking elements, closure under taking elements and subsets, closure under not increasing rank, and so forth, to closure under everything and giving rise to a linear structure of degrees of existence requirement. If a sentence is valid in the universe, the domain that corresponds to the highest closure degree, then one may ask how much closure of the universe is actually required by its validity. Using this apparatus, one can evaluate the axioms of $Z F C$, and the result is a refinement of the standard view about these axioms. For example, the axiom of regularity has no existential power, while the instances of replacement, in general, have strong existence requirements.

Received May 10, 2012; accepted June 11, 2012

2010 Mathematics Subject Classification: Primary 03E30

Keywords: existence axioms, foundations of set theory

(C) 2014 by University of Notre Dame 10.1215/00294527-2377896 
Although there is general agreement between the standard view and the results obtained in [1], there is also at least one point of divergence: the extensionality axiom. According to the standard view, extensionality has no existential import. The results presented in [1] show a different picture: the existential power of extensionality is indeed very weak, but it is not zero. The transitivity of a domain suffices to fulfill the existential demands of extensionality, but it is also required: if the domain is not transitive then extensionality may not hold. The following examples provide some proofs of (mathematically) interesting existential statements in which extensionality is used. (The first two were already hinted at in [1].)

Example 1 We have Cantor's proof that there exists a transcendental number: the set of algebraic numbers is countable, and the set of all real numbers is not. Therefore these two sets are different, and, by extensionality, there is a real number which is not algebraic. The use of extensionality can be eliminated; the diagonal method may be used to construct a transcendental number.

Example 2 Denote by $A$ the set of orderings in $\wp(\wp(\omega))$, and denote by $B$ the set of well-orderings in $\wp(\wp(\omega))$. If $A \neq B$, then there is an ordering in $\wp(\wp(\omega))$ which is not a well-ordering in $\wp(\wp(\omega))$. This is a theorem of $Z F$; it is almost an instance of extensionality, and $Z F C$ cannot provide an abstraction term for this existential theorem (see Levy [2, p. 173]).

Example 3 If $\alpha$ is an ordinal and $\alpha \neq \beth_{\alpha}$, then there is an element in $V_{\alpha}$ which is not in $L_{\alpha}$. This is a theorem of $Z F C$ : assuming the hypothesis, it follows that $L_{\alpha}$ and $V_{\alpha}$ are not equipotent. Since $L_{\alpha} \subset V_{\alpha}$, there is, by extensionality, an element in $V_{\alpha}$ which is not in $L_{\alpha}$.

The examples above confirm that the existential power of extensionality is indeed very limited but not zero. This is a point of divergence from the standard view, and there may be other points of divergence. According to the results in the first part, the axiom of the empty set is not different from the assertion $\exists x(x=x)$ : it excludes the empty domain, but it makes no further requirement for nonempty domains. The standard view seems not to equate these two assertions. The axiom of infinity is another possible point of disagreement. One could say that the classification in [1] overestimates the existence requirement of the axiom of infinity; it is in fact an existence assertion but may not be as strong as indicated by the classification presented in [1].

The disagreement with the standard view is not in itself a great problem; it was already pointed out in [1] that one of the defects of the standard view is that it lacks justification for its distinctions. Moreover, the standard view is not accurate or stable enough so that a comparison can be made to the theory developed in [1]. However, the analysis presented there is supposed not to have the same defects of the standard view and must be justified on solid grounds. This is one of the purposes of the present article.

The productivity of a valid sentence is classified according to seven linearly ordered degrees of existence requirement: $0,1,2,3,4,4 \omega, 5$. These are the absolute degrees of existence requirement: they measure the amount of closure (in a natural hierarchy of closure properties) that is sufficient for the validity of a sentence in a domain. The fact that the productivity of a sentence is a semantic notion, and must be evaluated by the semantic behavior of the sentence, implies that the productivity 
of a sentence is stable under logical deduction. In order to evaluate the classification of the axioms according to the absolute degrees obtained in [1], one could (i) enrich the structure of absolute degrees in a way that is coherent with the notion of productivity, (ii) reclassify the axioms according to the new structure, and (iii) see if the old classification persists in the new structure. The simple relative degrees defined in Section 2, Definition 23, form an enriched structure coherent with the notion of productivity, and Theorem 20 shows that the old classification of the axioms persists in the new structure, with the exception of the axiom of infinity.

\section{Relative Degrees}

Let $T^{*}$ be the theory obtained from $Z F C$ by the introduction of unquantifiable class variables, as described in [2] (in the first chapter), and by adding the appropriate relation and function symbols. $T^{*}$ is a conservative extension of $Z F C$. Let $\varphi(I)$ be a formula in $T^{*}$, in which $I$ is a class variable, and such that there are no free variables in $\varphi(I)$ besides $I$. Furthermore, $\varphi(I)$ may contain introduced symbols of $T^{*}$, such as the constant $\omega$. One may think of $\varphi(I)$ as a context, or a condition on the interpretations $I$, and the degrees of existence requirement in [1] can be relativized to this context: just relativize each item in the relevant definition to the context $\varphi(I)$. For example, the sentence $A$ is said to admit degree 1 of existence requirement relative to the context $\varphi(I)$ if, under the assumption of $\varphi(I), A$ holds in every transitive $\in$-interpretation of $L(Z F)$. The following definition makes precise the notion of degree of existence requirement relative to a context.

Definition 4 Let $T^{*}$ be the theory described above, and let $\varphi(I)$ be a context in $T^{*}$. The sentence $A$ in $L(Z F)$ is said to admit the following relative degrees of existence requirement:

- degree 0 of existence requirement relative to the context $\varphi(I)$ in $T^{*}$ if, under the assumption of $\varphi(I)$, the sentence $A$ holds in every (nonempty) $\in$ interpretation of $L(Z F)$ in $T$, that is, if

$$
T^{*} \vdash \varphi(I) \wedge \exists x(x \in I) \rightarrow A^{I} ;
$$

- degree 1 of existence requirement relative to the context $\varphi(I)$ in $T^{*}$ if, under the assumption of $\varphi(I)$, the sentence $A$ holds in every transitive $\in$-interpretation of $L(Z F)$ in $T$, that is, if

$$
T^{*} \vdash \varphi(I) \wedge \exists x(x \in I) \wedge \forall x \forall y(x \in I \wedge y \in x \rightarrow y \in I) \rightarrow A^{I} ;
$$

- degree 2 of existence requirement relative to the context $\varphi(I)$ in $T^{*}$ if, under the assumption of $\varphi(I)$, the sentence $A$ holds in every supertransitive $\in$-interpretation of $L(Z F)$ in $T$, that is, if

$$
T^{*} \vdash \varphi(I) \wedge \exists x(x \in I) \wedge \forall x \forall y(x \in I \wedge(y \in x \vee y \subset x) \rightarrow y \in I) \rightarrow A^{I} ;
$$

- degree 3 of existence requirement relative to the context $\varphi(I)$ in $T^{*}$ if, under the assumption of $\varphi(I)$, the sentence $A$ holds in every $\in$-interpretation of $L(Z F)$ in $T$ that is a level $V_{\alpha}$, that is, if

$$
T^{*} \vdash \varphi(I) \wedge \exists x(x \in I) \wedge \operatorname{Ord}(\alpha) \wedge \forall x\left(x \in I \leftrightarrow x \in V_{\alpha}\right) \rightarrow A^{I},
$$

in which $\operatorname{Ord}(\alpha)$ stands for " $\alpha$ is an ordinal"; 
- degree 4 of existence requirement relative to the context $\varphi(I)$ in $T^{*}$ if, under the assumption of $\varphi(I)$, the sentence $A$ holds in every $\in$-interpretation of $L(Z F)$ in $T$ that is a level $V_{\alpha}$ for $\alpha$ a limit ordinal, that is, if $T^{*} \vdash \varphi(I) \wedge \exists x(x \in I) \wedge \operatorname{LimOrd}(\alpha) \wedge \forall x\left(x \in I \leftrightarrow x \in V_{\alpha}\right) \rightarrow A^{I}$,

in which $\operatorname{LimOrd}(\alpha)$ stands for " $\alpha$ is a limit ordinal";

- degree $4 \omega$ of existence requirement relative to the context $\varphi(I)$ in $T^{*}$ if, under the assumption of $\varphi(I)$, the sentence $A$ holds in every $\in$-interpretation of $L(Z F)$ in $T$ that is a level $V_{\alpha}$ for $\alpha$ a limit ordinal greater than $\omega$, that is, if

$$
T^{*} \vdash \varphi(I) \wedge \operatorname{LimOrd}(\alpha) \wedge \omega<\alpha \wedge \forall x\left(x \in I \leftrightarrow x \in V_{\alpha}\right) \rightarrow A^{I} ;
$$

- degree 5 of existence requirement relative to the context $\varphi(I)$ in $T^{*}$ if, under the assumption of $\varphi(I)$, the sentence $A$ holds in the identity interpretation $\mathbf{V}$ of $L(Z F)$ in $T$, that is, if

$$
T^{*} \vdash \varphi(I) \wedge \forall x(x \in I) \rightarrow A^{I} .
$$

Remark 5 All clauses in Definition 4, with the exception of the clause for degree 0 , are of the form $T^{*} \vdash \varphi(I) \wedge C^{d}(I) \rightarrow A^{I}$, in which $C^{d}(I)$ depends on the degree $d$ and expresses the corresponding closure property of $I$. Definition 4 is slightly different from the corresponding definition in [1]: in the former the clauses are formulas in the extended language of $T^{*}$, while in the latter the clauses are schemas. It is assumed, tacitly, that $\mathbf{V}$ has all closure properties, that is, the formula $C^{d}(I)$ is such that for each $d \in\{0,1,2,3,4,4 \omega, 5\}, T^{*} \vdash C^{d}(\mathbf{V})$.

Remark 6 The absolute productivity can be recovered in Definition 4 by a tautological context such as $\forall x(x=x)$ : if the sentence $A$ in $L(Z F)$ admits degree $d$ relative to $\forall x(x=x)$ according to Definition 4 , then $A$ admits (absolute) degree $d$ according to the original definition in [1]. This is an immediate corollary of the conservation theorem [2, Theorem I.4.6], except that for degrees $3,4,4 \omega$, and 5 one must consider a slight reformulation of the original definition in [1] and remember that in the original definition the hypothesis $\exists x(x \in I)$ is implicit. ${ }^{1}$ Moreover, if the sentence $A$ is $B \rightarrow C$, then $A$ admits (absolute) degree $d$ of existence requirement if and only if $C$ admits degree $d$ of existence requirement relative to the context $B^{I}$.

Remark 7 All the results in [1, Section 2] admit a relativized version: the relative degrees are linearly ordered by strength; a sentence $A$ in $L(Z F)$ admits a relative degree if and only if $T^{*} \vdash \varphi(I) \rightarrow A$; if $\vdash A \rightarrow B$, and $A$ admits degree $d$ relative to $\varphi(I)$, then $B$ also admits degree $d$ relative to $\varphi(I)$; and so forth. The convention for contexts makes clear that the class variable $I$ is the only variable that may occur free in a context. When there is no risk of ambiguity, the variable $I$ will not be indicated, and a context will be denoted simply by $\varphi, \varphi^{\prime}$, and so forth. If $\varphi \rightarrow A$ is a theorem of $T^{*}$, then the least degree of existence requirement relative to $\varphi$ admitted by $A$ is denoted by $r(A \mid \varphi)$. If $r(A \mid \varphi)$ is defined, then it is called the existence requirement of $A$ relative to $\varphi$.

Remark 8 Since one can consider the degree of existence requirement of the sentence $A$ relative to the context $A$, Definition 4 also subsumes part of the analysis of stronger theories set forth in [1].

Let $P$ denote the set of all pairs $(d, \varphi)$, in which $d$ is an absolute degree and $\varphi$ is a context in $T$. There are two natural preorderings in $P$ related to Definition 4: 
(1) $(d, \varphi) \leq_{1}\left(d^{\prime}, \varphi^{\prime}\right)$ if and only if $d \preceq d^{\prime}$ and $T^{*} \vdash\left(\varphi^{\prime} \wedge C^{d^{\prime}}(I)\right) \rightarrow \varphi$;

(2) $(d, \varphi) \leq_{2}\left(d^{\prime}, \varphi^{\prime}\right)$ if and only if $d \preceq d^{\prime}$ and $T^{*} \vdash \varphi^{\prime} \rightarrow \varphi$.

It follows by definition that $\leq_{2} \subset \leq_{1}$. Lemma 9 shows that $P$ is preordered by strength of relative productivity by both relations $\leq_{1}$ and $\leq_{2}$.

Lemma 9 Consider $A$ a sentence in $L(Z F)$. If $(d, \varphi) \leq_{1}\left(d^{\prime}, \varphi^{\prime}\right)$ or $(d, \varphi) \leq_{2}$ $\left(d^{\prime}, \varphi^{\prime}\right)$, and if $A$ admits degree $d$ of existence requirement relative to the context $\varphi$ in $T^{*}$, then $A$ admits degree $d^{\prime}$ of existence requirement relative to the context $\varphi^{\prime}$ in $T^{*}$.

Proof Suppose that $(d, \varphi) \leq_{1}\left(d^{\prime}, \varphi^{\prime}\right)$. In this case, $T^{*} \vdash\left(\varphi^{\prime} \wedge C^{d^{\prime}}(I)\right) \rightarrow \varphi$ and $d \preceq d^{\prime}$. Since $T^{*} \vdash C^{d^{\prime}}(I) \rightarrow C^{d}(I)$, if $A$ admits degree $d$ of existence requirement relative to the context $\varphi$ in $T^{*}$, then $T^{*} \vdash \varphi \wedge C^{d}(I) \rightarrow A^{I}$. It follows that $A$ admits degree $d^{\prime}$ of existence requirement relative to the context $\varphi^{\prime}$ in $T^{*}$.

If $(d, \varphi) \leq_{2}\left(d^{\prime}, \varphi^{\prime}\right)$, then $T^{*} \vdash \varphi^{\prime} \rightarrow \varphi$. Now the argument above shows that $A$ admits degree $d^{\prime}$ of existence requirement relative to the context $\varphi^{\prime}$ in $T^{*}$.

Consider the equivalence relations defined below:

$(d, \varphi) \equiv_{i}\left(d^{\prime}, \varphi^{\prime}\right)$ if and only if $(d, \varphi) \leq_{i}\left(d^{\prime}, \varphi^{\prime}\right)$ and $\left(d^{\prime}, \varphi^{\prime}\right) \leq_{i}(d, \varphi), i=1,2$.

Although $\equiv_{2} \subset \equiv_{1}$, these equivalence relations are different. In fact, if $\varphi$ is the extensionality axiom and $\varphi^{\prime}$ is a tautology, then $(1, \varphi) \equiv_{1}\left(1, \varphi^{\prime}\right)$, but $(1, \varphi)$ and $\left(1, \varphi^{\prime}\right)$ are not equivalent according to $\equiv_{2} \cdot{ }^{2}$ Denote by $Q_{i}$ the quotient of $P$ by the equivalence relation $\equiv_{i}$, for $i=1,2$. The ordering induced by $\leq_{i}$ in $Q_{i}$ is denoted by the same symbol $\leq_{i}$, for $i=1,2$.

Proposition 10 The set $Q_{2}$ ordered by $\leq_{2}$ is a lattice and is isomorphic to the direct product of the lattice $(D, \prec)$ of absolute degrees with $B_{0}^{\mathrm{op}}\left(T^{*}\right)$, the 0 th Lindenbaum-Tarski algebra of $T^{*}$ with the opposite ordering.

Proof Let $\left|B_{0}^{\mathrm{op}}\left(T^{*}\right)\right|$ denote the domain of $B_{0}^{\mathrm{op}}\left(T^{*}\right)$. The map

$$
F: P \longrightarrow D \times\left|B_{0}^{\mathrm{op}}\left(T^{*}\right)\right|, \quad(d, \varphi) \mapsto(d,[\varphi])
$$

is surjective and is a homomorphism of preordered sets. The required isomorphism is obtained by passing $F$ to the quotient.

Proposition 10 shows that $Q_{2}$ is not quite adequate to capture the notion of relative degrees. The structure of relative degrees is supposed to come from the interaction between the structure of absolute degrees on one side and the structure of contexts on the other. Proposition 10 shows that in $Q_{2}$ there is little, if any, interaction. The structure of $Q_{1}$ is much more interesting and more adequately captures the interaction between absolute degrees and contexts.

Proposition 11 The set $Q_{1}$ ordered by $\leq_{1}$ is a lattice. In this lattice sup and inf, $\top$ and $\perp$ are given by the following formulas:

$$
\begin{aligned}
& \sup \left([(d, \varphi)]_{1},\left[\left(d^{\prime}, \varphi^{\prime}\right)\right]_{1}\right)=\left[\left(\max \left(d, d^{\prime}\right), \varphi \wedge \varphi^{\prime}\right)\right]_{1}, \\
& \inf \left([(d, \varphi)]_{1},\left[\left(d^{\prime}, \varphi^{\prime}\right)\right]_{1}\right) \\
& \quad=\left[\left(\min \left(d, d^{\prime}\right),\left(\varphi \vee \varphi^{\prime}\right) \wedge\left(\varphi \vee C^{d^{\prime}}(I)\right) \wedge\left(\varphi^{\prime} \vee C^{d}(I)\right)\right]_{1},\right. \\
& \top=[(5, \varphi \wedge \neg \varphi)]_{1}, \\
& \perp=[(0, \varphi \vee \neg \varphi)]_{1},
\end{aligned}
$$

in which $[(d, \varphi)]_{1}$ denote the class of $(d, \varphi)$ according to $\equiv_{1}$. 
Proof This is straightforward. Suppose that $(d, \varphi) \leq_{1}(e, \psi)$ and that $\left(d^{\prime}, \varphi^{\prime}\right) \leq_{1}$ $(e, \psi)$. This means that $\max \left(d, d^{\prime}\right) \preceq e$ and that

$$
T^{*} \vdash \psi \wedge C^{e}(I) \rightarrow \varphi \quad \text { and } \quad T^{*} \vdash \psi \wedge C^{e}(I) \rightarrow \varphi^{\prime} .
$$

Therefore, $T^{*} \vdash \psi \wedge C^{e}(I) \rightarrow \varphi \wedge \varphi^{\prime}$ and $\left(\max \left(d, d^{\prime}\right), \varphi \wedge \varphi^{\prime}\right) \leq_{1}(e, \psi)$. Since $(d, \varphi) \leq_{1}\left(\max \left(d, d^{\prime}\right), \varphi \wedge \varphi^{\prime}\right)$ and $\left(d^{\prime}, \varphi^{\prime}\right) \leq_{1}\left(\max \left(d, d^{\prime}\right), \varphi \wedge \varphi^{\prime}\right)$, it follows that

$$
\sup \left([(d, \varphi)]_{1},\left[\left(d^{\prime}, \varphi^{\prime}\right)\right]_{1}\right)=\left[\left(\max \left(d, d^{\prime}\right), \varphi \wedge \varphi^{\prime}\right)\right]_{1} .
$$

In order to establish the formula for inf, suppose that $(e, \psi) \leq_{1}(d, \varphi)$ and that $(e, \psi) \leq_{1}\left(d^{\prime}, \varphi^{\prime}\right)$. This means that $e \preceq \min \left(d, d^{\prime}\right)$ and that

$$
T^{*} \vdash \varphi \wedge C^{d}(I) \rightarrow \psi \quad \text { and } \quad T^{*} \vdash \varphi^{\prime} \wedge C^{d^{\prime}}(I) \rightarrow \psi .
$$

Therefore, $T^{*} \vdash\left(\varphi \wedge C^{d}(I)\right) \vee\left(\varphi^{\prime} \wedge C^{d^{\prime}}(I)\right) \rightarrow \psi$. Equivalently,

$$
T^{*} \vdash\left(\varphi \vee \varphi^{\prime}\right) \wedge\left(\varphi \vee C^{d^{\prime}}(I)\right) \wedge\left(\varphi^{\prime} \vee C^{d}(I)\right) \wedge\left(C^{d}(I) \vee C^{d^{\prime}}(I)\right) \rightarrow \psi
$$

The inequality

$$
(e, \psi) \leq_{1}\left(\min \left(d, d^{\prime}\right),\left(\varphi \vee \varphi^{\prime}\right) \wedge\left(\varphi \vee C^{d^{\prime}}(I)\right) \wedge\left(\varphi^{\prime} \vee C^{d}(I)\right)\right)
$$

follows from the fact that $T^{*} \vdash\left(C^{d}(I) \vee C^{d^{\prime}}(I)\right) \leftrightarrow C^{\min \left(d, d^{\prime}\right)}(I)$. Since

$$
T^{*} \vdash\left(\varphi \vee \varphi^{\prime}\right) \wedge\left(\varphi \vee C^{d^{\prime}}(I)\right) \wedge\left(\varphi^{\prime} \vee C^{d}(I)\right) \wedge C^{\min \left(d, d^{\prime}\right)}(I) \rightarrow \varphi
$$

and

$$
T^{*} \vdash\left(\varphi \vee \varphi^{\prime}\right) \wedge\left(\varphi \vee C^{d^{\prime}}(I)\right) \wedge\left(\varphi^{\prime} \vee C^{d}(I)\right) \wedge C^{\min \left(d, d^{\prime}\right)}(I) \rightarrow \varphi^{\prime},
$$

it follows that

$\inf \left([(d, \varphi)]_{1},\left[\left(d^{\prime}, \varphi^{\prime}\right)\right]_{1}\right)=\left[\left(\min \left(d, d^{\prime}\right),\left(\varphi \vee \varphi^{\prime}\right) \wedge\left(\varphi \vee C^{d^{\prime}}(I)\right) \wedge\left(\varphi^{\prime} \vee C^{d}(I)\right)\right)\right]_{1}$.

Using the formulas for sup and inf, it is easy to prove the formulas for $\top$ and $\perp$.

The lattices $\left(Q_{1}, \leq_{1}\right)$ and $\left(Q_{2}, \leq_{2}\right)$ provide two different notions of relative degrees of productivity. It has already been remarked that in $\left(Q_{2}, \leq_{2}\right)$ there is little interaction between the structure of absolute degrees and the structure of contexts, while in $\left(Q_{1}, \leq_{1}\right)$ there is a stronger interaction. Later in this section, a third natural preordering $\leq_{0}$ in $P$ will be introduced, together with the associated partially ordered quotient $\left(Q_{0}, \leq_{0}\right)$, giving rise to a third notion of relative degree.

One may ask what relative degrees, in one sense or another, are admitted by a sentence in $L(Z F)$. Of course, for any sentence $A$ there is a context $\varphi$ such that $A$ admits degree 0 of existence requirement relative to $\varphi$; just take $\varphi$ to be $A \wedge(I=\mathbf{V})$, or just $A^{I}$. This means that $A$ admits all relative degrees $\left[\left(d, A^{I}\right)\right]$, for $d=0, \ldots, 5$, no matter which specific notion of relative degree is used. Therefore, the class of all contexts gives rise to a trivial notion of reducibility: everything is reducible to zero in this class. Definition 12 below specifies a subclass of contexts that corresponds to a nontrivial notion of reducibility, and that will be valuable in the evaluation of the productivity of the axioms of $Z F C$.

Definition 12 The formula $\varphi$ is called a simple context if it is a context of the form

$$
\exists x_{1} \cdots \exists x_{n}\left(\varphi\left(x_{1}, \ldots, x_{n}\right) \wedge x_{1} \in I \wedge \cdots \wedge x_{n} \in I\right),
$$


in which it is assumed that $n$ is a natural number (possibly zero), all free variables of $\varphi$ are included in the list $x_{1}, \ldots, x_{n}$ ( $I$ does not occur in $\varphi$ ), and, assuming the consistency of $Z F, Z F C \nvdash \neg \exists x_{1} \cdots \exists x_{n} \varphi$.

Remark 13 The context $\varphi$ of the form

$$
\exists x_{1} \cdots \exists x_{n}\left(\varphi\left(x_{1}, \ldots, x_{n}\right) \wedge x_{1} \in I \wedge \cdots \wedge x_{n} \in I\right)
$$

can appear as

$$
\exists \bar{x}(\varphi(\bar{x}) \wedge \bar{x} \in I) .
$$

If more than one context is under consideration, for example, $\varphi$ and $\varphi^{\prime}$, then they can appear as

respectively.

$$
\exists \bar{x}(\varphi(\bar{x}) \wedge \bar{x} \in I) \quad \text { and } \quad \exists \bar{y}\left(\varphi^{\prime}(\bar{y}) \wedge \bar{y} \in I\right),
$$

Remark 14 The condition $Z F C \nvdash \neg \exists \bar{x} \varphi$ in Definition 12 implies that $\exists \bar{x} \varphi$ is consistent with $Z F C$, and, by the conservation theorem [2, Theorem I.4.6], it is equivalent to $T^{*} \nvdash \neg \exists \bar{x} \varphi$.

The notion of simple context will be used to evaluate the classification of the axioms according to the absolute degrees obtained in [1]. In the introduction, it was remarked that in order to evaluate that classification one could (i) enrich the structure of absolute degrees in a way that is coherent with the notion of productivity, (ii) reclassify the axioms according to the new structure, and (iii) see if the old classification persists in the new structure. Definition 12 is the first step toward (i), (ii), and (iii).

Before doing so, however, it should be remarked that the notion of simple context is coherent with the notion of productivity; in fact, productivity is the capacity of producing new sets from given sets in nonempty domains. This existential power should not be lost by the restriction to nonempty domains that contain some sets $x_{1}, \ldots, x_{n}$, satisfying some consistent property. Simple contexts cannot raise the closure degree: satisfying a simple context is not sufficient to guarantee that a domain is transitive, or that a transitive domain is supertransitive, and so forth. ${ }^{3}$ The rest of this section is devoted to the classification of the axioms of $Z F C$ according to this structure. In the end of the section, four results on the structure of relative degrees of the form $[(d, \varphi)]_{1}$, where $\varphi$ is simple, are included.

Definition 15 Suppose that the sentence $A$ admits a degree of existence requirement. The sentence $A$ is simply reducible if there is a simple context $\varphi$ such that $r(A \mid \varphi) \prec r(A)$. The sentence $A$ is simply reducible to degree $d$ if there is a simple context $\varphi$ such that $A$ admits degree $d$ of existence requirement relative to $\varphi$ and $d \prec r(A)$. The sentence $A$ is simply irreducible if it is not simply reducible.

Remark 16 If the sentence $A$ is such that $0 \prec r(A)$, then $A$ is simply reducible if and only if $A$ is simply reducible to the degree immediately preceding $r(A)$. In case it is relevant to indicate both the degree and context that gives the reduction, one may say that $A$ is simply reducible to degree $d$ under $\varphi$.

In [1, Part I], one encounters the following result: if $A$ admits a degree of existence requirement and $A$ is of the form $\forall x_{1} \cdots \forall x_{n} B$, in which all quantifiers in $B$ are bounded, then $A$ admits degree 1. Proposition 17 complements this result: without alternating unbounded quantifiers one cannot obtain simply irreducible existence requirements greater than 1 . 
Proposition 17 If $A$ is a sentence of $L(Z F), Z F C \nvdash \neg A$ and $A$ is of the form $\exists x_{1} \cdots \exists x_{n} B$, in which all quantifiers in $B$ are bounded, then there is a simple context $\varphi$ such that $A$ admits degree 1 relative to $\varphi$.

Proof Take $\varphi$ to be the context $\exists x_{1} \cdots \exists x_{n}\left(B \wedge x_{1} \in I \wedge \cdots \wedge x_{n} \in I\right)$. The closure condition $C^{1}(I)$ implies that $A^{I} \leftrightarrow \varphi$.

The aim of the following results is to show that the axioms of $Z F C-\operatorname{Inf}(Z F C$ minus the axiom of infinity) are simply irreducible and that the axiom of infinity is simply reducible to degree 1 .

Lemma 18 If $\varphi$ is a simple context, then there is a natural number $N$ such that $T^{*} \nvdash \varphi \rightarrow N \in I$.

Proof Suppose that $\varphi$ is

$$
\exists x_{1} \cdots \exists x_{n}\left(\varphi\left(x_{1}, \ldots, x_{n}\right) \wedge x_{1} \in I \wedge \cdots \wedge x_{n} \in I\right) .
$$

Suppose, on the contrary, that for each $N$ it holds that $T^{*} \vdash \varphi \rightarrow N \in I$. In this case, for each $N$,

$$
T^{*} \vdash \exists \bar{x}\left(\varphi(\bar{x}) \wedge \bar{x} \in\left\{y_{1}, \ldots, y_{n}\right\}\right) \rightarrow N \in\left\{y_{1}, \ldots, y_{n}\right\} .
$$

Since $T^{*} \vdash \varphi(\bar{y}) \rightarrow \exists \bar{x}\left(\varphi(\bar{x}) \wedge \bar{x} \in\left\{y_{1}, \ldots, y_{n}\right\}\right)$, it follows that for each $N$, $T^{*} \vdash \varphi(\bar{y}) \rightarrow N \in\left\{y_{1}, \ldots, y_{n}\right\}$. In particular,

$$
T^{*} \vdash \varphi(\bar{y}) \rightarrow\left(0 \in\left\{y_{1}, \ldots, y_{n}\right\} \wedge \cdots \wedge S^{n} 0 \in\left\{y_{1}, \ldots, y_{n}\right\}\right),
$$

and hence $T^{*} \vdash \neg \varphi(\bar{y})$, contradicting the hypothesis that $\varphi$ is a simple context.

Remark 19 The notation $S^{n} 0$ is used in order to distinguish between (the metatheoretical number) $n$ and the corresponding number in $T^{*}$. The symbol $S$ denotes the successor function symbol. The proof of Lemma 18 shows that if $n$ is the number of free variables in $\varphi$, then there is a number $N \leq S^{n} 0$ such that $T^{*} \nvdash \varphi \rightarrow N \in I$. Moreover, $T^{*}$ cannot prove that the simple context $\varphi$ implies that $I$ is infinite.

Theorem 20 The axioms of $Z F C$ - Inf are not simply reducible. The axiom of infinity is simply reducible; if $A$ is

$$
\exists x(\exists y \in x(\forall z(z \notin y)) \wedge \forall y \in x(\exists z \in x(\forall w(w \in z \leftrightarrow w \in y \vee w=y))))
$$

and $\varphi$ is the simple context $\exists x(x=\omega \wedge x \in I)$, then $r(A \mid \varphi)=1$.

Proof Clearly, if $A$ admits degree 0 of existence requirement, then $A$ is simply irreducible. Therefore, only the productive axioms are considered.

Beginning with the axiom of infinity, the sentence $A$ in the statement is of the form $\exists x B$. Fix $I$, and suppose that $\varphi \wedge C^{1}(I)$, in which $\varphi$ is the simple context $\exists x(x=\omega \wedge x \in I)$. Since $\omega \in I$ and $I$ is transitive, it follows that $n \in I$, for any natural number $n$. It is now clear that $B_{x}[\omega]^{I}$ holds. From this and the fact that $\omega \in I$, it follows that $A^{I}$ holds. The proof of Lemma 18 shows that a simple context cannot imply that $I$ is infinite, and hence the axiom of infinity is not simply reducible to degree 0 .

There are instances of the axiom of replacement that are not simply reducible. In fact, it is a theorem of $Z F C$ that every well-ordered set is isomorphic to a unique ordinal, and hence this is a theorem of a finite part $\Gamma$ of $Z F C$. Let $A_{1}, \ldots, A_{k}$ be the 
instances of replacement in $\Gamma$. Suppose that, for a fixed $I$, there is a simple context $\varphi$ of the form

$$
\exists x_{1} \cdots \exists x_{n}\left(\varphi\left(x_{1}, \ldots, x_{n}\right) \wedge x_{1} \in I \wedge \cdots \wedge x_{n} \in I\right)
$$

such that $T^{*}$ proves all instances of replacement $A_{1}, \ldots, A_{k}$ relativized to $I$, under the hypothesis of $\varphi \wedge C^{4 \omega}(I)$. Since

$$
T^{*} \vdash \varphi\left(y_{1}, \ldots, y_{n}\right) \rightarrow \exists x_{1} \ldots \exists x_{n} \varphi\left(x_{1}, \ldots, x_{n}\right)
$$

and $T^{*} \vdash \exists \alpha>\omega\left(\operatorname{LimOrd}(\alpha) \wedge y_{1} \in V_{\alpha+\alpha} \wedge \cdots \wedge y_{n} \in V_{\alpha+\alpha}\right)$, it follows that

$$
T^{*} \vdash \varphi(\bar{y}) \rightarrow \exists \alpha \exists \bar{x}\left(\varphi(\bar{x}) \wedge \bar{x} \in V_{\alpha+\alpha}\right) \wedge C^{4 \omega}\left(V_{\alpha+\alpha}\right) .
$$

Therefore, $T^{*} \vdash \varphi(\bar{y}) \rightarrow \exists \alpha>\omega\left(\operatorname{LimOrd}(\alpha) \wedge A_{i}^{V_{\alpha+\alpha}}\right)$, for all instances $A_{i}$, $i=1, \ldots, k$, of replacement. But $\alpha+\alpha$ is a limit ordinal greater than $\omega$, and all axioms of $Z C$ hold in $V_{\alpha+\alpha}$. Since $2 \times \alpha$ ordered lexicographically is a well-ordered set in $V_{\alpha+\alpha}$ and $2 \times \alpha$ is isomorphic to $\alpha+\alpha$, it follows that $T^{*}+\exists \bar{y} \varphi(\bar{y})$ proves that there is an ordinal $\alpha$ such that $\alpha+\alpha \in V_{\alpha+\alpha}$, contradicting the hypothesis that $\varphi$ is a simple context.

Similarly, the other axioms are not simply reducible. As shown in the argument for the nonreducibility of replacement, a simple context $\varphi$ requires only the existence in $I$ of some sets $x_{1}, \ldots, x_{n}$ satisfying $\varphi$. The axiom $A$, such that $0 \prec r(A)$, is simply reducible to the degree immediately preceding $r(A)$ under $\varphi$ if $A$ holds in any domain $I$ containing $x_{1}, \ldots, x_{n}$ and such that $C^{d}(I)$. For extensionality this is impossible; in this case, $r(A)=1$, and it suffices to take $I=\left\{x_{1}, \ldots, x_{n}, y, z\right\}$ such that $y \neq z$ and for every $w \in y \cup z, w \notin I$. For separation, $r(A)=2$ and it suffices to take $I$ to be the transitive closure of $\left\{\left\{x_{1}, \ldots, x_{n}\right\}\right\}$. In fact, if $x_{i}$ is of maximal rank, then $\left\{x_{i}\right\}$ is not in $I$. For the power set (and pairing), $r(A)=4$ and it suffices to take $I=V_{\alpha+1}$ such that $\alpha$ is greater than the rank of $\left\{x_{1}, \ldots, x_{n}\right\}$. For the choice set (and union), $r(A)=3$. Fix $\alpha+1$ greater than the rank $\left\{x_{1}, \ldots, x_{n}\right\}$, and take $I=V_{\alpha+1} \cup\{\{\alpha\},\{\{\alpha\}\},\{\{\{\alpha\}\}\},\{\{\alpha\},\{\{\alpha\}\}\}\}$. The set $\{\alpha,\{\alpha\}\}$ is not in $I$.

Remark 21 There are, in the literature, formulations of the axiom of infinity that are not logically equivalent. For example, the version of this axiom in [2] is different from the sentence evaluated above. One could also simply assert that there is an infinite set. However, all of these sentences are simply reducible to degree 1 . If $\varphi$ is the simple context $\exists x\left(x=V_{\omega} \wedge x \in I\right)$, then the axiom of infinity, as formulated in [2], admits degree 1 relative to $\varphi$. Furthermore, if $I$ is transitive and $\omega \in I$, then $\omega$ is an infinite set in $I$.

Remark 22 If $A$ admits degree $4 \omega$ of existence requirement relative to a simple context of the form $\exists \bar{x}(\varphi(\bar{x}) \wedge \bar{x} \in I)$, then $A$ also admits degree 4 relative to the simple context $\exists \bar{x} \exists y(\varphi(\bar{x}) \wedge y=\omega \wedge \bar{x} \in I \wedge y \in I)$.

Theorem 20 shows that, with the exception of the axiom of infinity, the classification achieved in [1] persists in the structure of simple relative degrees. This theorem also shows that the axiom of infinity makes very weak existential demands on domains, except for requiring that $\omega$ is in the domain. This seems to be in agreement with the intuitive understanding of this axiom.

The previous results regarding simple reducibility suggest the identification of strongly productive assertions with sentences that do not admit degree 1 and that are not simply reducible to degree 1 . This seems to be a reasonable definition of the 
intuitive notion of strongly productive assertion. In this sense, the strongly productive axioms of $Z F C$ are separation, power set, choice, replacement, union, and pairing.

Definition 23 The classes $[(d, \varphi)]_{1}$, in which $\varphi$ is a simple context, are called simple relative degrees, and the set of all such classes is denoted by $S$. According to Proposition 24 below, $S$ with the induced ordering $\leq_{1}$ is an upper semilattice.

This section ends with the following results on the structure of $\left(S, \leq_{1}\right)$.

Proposition 24 Denote by $[(d, \varphi)]_{1}$ and $[(d, \varphi)]_{2}$ the classes of $(d, \varphi)$ according to $\equiv_{1}$ and $\equiv_{2}$, respectively.

(1) The classes $[(d, \varphi)]_{2}$, in which $\varphi$ is a simple context, form a sublattice of $\left(Q_{2}, \leq_{2}\right)$.

(2) The classes $[(d, \varphi)]_{1}$, in which $\varphi$ is a simple context, form an upper subsemilattice of $\left(Q_{1}, \leq_{1}\right)$ with the least element $\perp$ and such that for any two elements there is a lower bound in the semilattice.

Proof (1): Suppose that $\varphi$ and $\varphi^{\prime}$ are simple contexts of the form

$$
\exists x_{1} \cdots \exists x_{n}\left(\varphi\left(x_{1}, \ldots, x_{n}\right) \wedge x_{1} \in I \wedge \cdots \wedge x_{n} \in I\right)
$$

and

$$
\exists y_{1} \cdots \exists y_{m}\left(\varphi^{\prime}\left(y_{1}, \ldots, y_{m}\right) \wedge y_{1} \in I \wedge \cdots \wedge y_{m} \in I\right), \quad \text { respectively. }
$$

The conjunction $\varphi \wedge \varphi^{\prime}$ is logically equivalent to the simple context

$$
\exists x_{1} \cdots \exists x_{n} \exists y_{1} \cdots \exists y_{m}\left(\varphi\left(x_{1}, \ldots, x_{n}\right) \wedge \varphi^{\prime}\left(y_{1}, \ldots, y_{m}\right) \wedge x_{1} \in I \wedge \cdots \wedge y_{m} \in I\right) \text {. }
$$

Assuming that $I$ is nonempty, the disjunction $\varphi \vee \varphi^{\prime}$ is equivalent to the simple context

$\exists x_{1} \cdots \exists x_{n} \exists y_{1} \cdots \exists y_{m}\left(\left(\varphi\left(x_{1}, \ldots, x_{n}\right) \vee \varphi^{\prime}\left(y_{1}, \ldots, y_{m}\right)\right) \wedge x_{1} \in I \wedge \cdots \wedge y_{m} \in I\right)$.

(2): This follows from (1) and the fact that $\leq_{2} \subset \leq_{1}$.

The semilattice $S$ is an enrichment of the structure of absolute degrees that is coherent with the notion of productivity. The use of this enriched structure in the evaluation of the productivity of a sentence gives more accurate results.

Proposition 25 The semilattice $\left(S, \leq_{1}\right)$ is not a lattice.

Proof Suppose that $\varphi, \varphi^{\prime}$, and $\varphi^{\prime \prime}$ are simple contexts, that $T^{*} \nvdash \varphi^{\prime} \wedge C^{4 \omega}(I) \rightarrow \varphi$, and that $\left(0, \varphi^{\prime \prime}\right)$ is a lower bound for $(0, \varphi)$ and $\left(4 \omega, \varphi^{\prime}\right)$ according to $\leq_{1}$. In this setting, $T^{*} \nvdash \varphi^{\prime \prime} \rightarrow \varphi$.

From Lemma 18, it follows that there is a natural number $N$ such that $T^{*} \nvdash \varphi^{\prime \prime} \rightarrow$ $N \in I$. Now if $\psi$ is the simple context

$$
\exists \bar{x} \exists \bar{y} \exists z\left((\varphi(\bar{x}) \vee z=N) \wedge \varphi^{\prime \prime}(\bar{y}) \wedge \bar{x} \in I \wedge \bar{y} \in I \wedge z \in I\right),
$$

then $\left(0, \varphi^{\prime \prime}\right) \leq_{1}(0, \psi)$, and $(0, \psi)$ is still a lower bound for $(0, \varphi)$ and $\left(4 \omega, \varphi^{\prime}\right)$. Since $T^{*} \nvdash \varphi^{\prime \prime} \rightarrow \varphi$, it follows that $\left(0, \varphi^{\prime \prime}\right)$ and $(0, \psi)$ are not equivalent according to $\equiv_{1}$. It follows that $(0, \varphi)$ and $\left(4 \omega, \varphi^{\prime}\right)$ have no greatest lower bound in $S$.

Proposition 25 shows that $S$ is not a lattice. However, for each fixed $d$, the set of all classes $[(d, \varphi)]$ contained in $S$ is a closed subset of $\left(Q_{1}, \leq_{1}\right)$ under the operations of inf and sup. 
Proposition 26 The map $H: S \longrightarrow S$ described below is a homomorphism of semilattices:

- $[(d, \varphi)]_{1} \mapsto[(d, \varphi)]_{1}$, if d is not $4 \omega$;

- $[(4 \omega, \varphi)]_{1} \mapsto[(4, \exists \bar{x} \exists z(\varphi(\bar{x}) \wedge z=\omega \wedge \bar{x} \in I \wedge z \in I))]_{1}$, where $\varphi$ is $\exists \bar{x}(\varphi(\bar{x}) \wedge \bar{x} \in I)$.

Proof It is necessary to prove that $H$ preserves the supremum of two simple relative degrees. The only case that requires proof is the case in which at least one of the degrees is of the form $[(4 \omega, \varphi)]_{1}$.

Let $[(4 \omega, \varphi)]_{1}$ and $\left[\left(d, \varphi^{\prime}\right)\right]_{1}$ be simple relative degrees. By Proposition 11,

$$
\sup \left([(4 \omega, \varphi)]_{1},\left[\left(d, \varphi^{\prime}\right)\right]_{1}\right)=\left[\left(\max (4 \omega, d), \varphi \wedge \varphi^{\prime}\right)\right]_{1} .
$$

It is necessary to prove that

$$
\sup \left(H\left([(4 \omega, \varphi)]_{1}\right), H\left(\left[\left(d, \varphi^{\prime}\right)\right]_{1}\right)\right)=H\left(\left[\left(\max (4 \omega, d), \varphi \wedge \varphi^{\prime}\right)\right]_{1}\right) .
$$

If $d \prec 4 \omega$, then equation (1) above becomes

$$
\begin{aligned}
& \sup \left([(4, \exists \bar{x} \exists z(\varphi(\bar{x}) \wedge z=\omega \wedge \bar{x} \in I \wedge z \in I))]_{1},\left[\left(d, \varphi^{\prime}\right)\right]_{1}\right) \\
& \quad=\left[\left(4, \exists \bar{x} \exists \bar{y} \exists z\left(\varphi(\bar{x}) \wedge \varphi^{\prime}(\bar{y}) \wedge z=\omega \wedge \bar{x} \in I \wedge \bar{y} \in I \wedge z \in I\right)\right)\right]_{1},
\end{aligned}
$$

where $\varphi$ is $\exists \bar{x}(\varphi(\bar{x}) \wedge \bar{x} \in I)$ and $\varphi^{\prime}$ is $\exists \bar{y}\left(\varphi^{\prime}(\bar{y}) \wedge \bar{y} \in I\right)$. In order to prove this equation, suppose that $(4, \exists \bar{x} \exists z(\varphi(\bar{x}) \wedge z=\omega \wedge \bar{x} \in I \wedge z \in I)) \leq_{1}(e, \psi)$ and $\left(d, \varphi^{\prime}\right) \leq{ }_{1}(e, \psi)$. Since $d \prec 4 \omega$, it follows that $4 \preceq e$. Furthermore,

$$
T^{*} \vdash \psi \wedge C^{e}(I) \rightarrow \exists \bar{x} \exists z(\varphi(\bar{x}) \wedge z=\omega \wedge \bar{x} \in I \wedge z \in I)
$$

and

$$
T^{*} \vdash \psi \wedge C^{e}(I) \rightarrow \varphi^{\prime}
$$

By the fact that $\varphi^{\prime}$ is $\exists \bar{y}\left(\varphi^{\prime}(\bar{y}) \wedge \bar{y} \in I\right)$, it follows that

$$
T^{*} \vdash \psi \wedge C^{e}(I) \rightarrow \exists \bar{x} \exists \bar{y} \exists z\left(\varphi(\bar{x}) \wedge \varphi^{\prime}(\bar{y}) \wedge z=\omega \wedge \bar{x} \in I \wedge \bar{y} \in I \wedge z \in I\right),
$$

and $\left(4, \exists \bar{x} \exists \bar{y} \exists z\left(\varphi(\bar{x}) \wedge \varphi^{\prime}(\bar{y}) \wedge z=\omega \wedge \bar{x} \in I \wedge \bar{y} \in I \wedge z \in I\right)\right) \leq_{1}(e, \psi)$. Clearly, $\left(4, \exists \bar{x} \exists \bar{y} \exists z\left(\varphi(\bar{x}) \wedge \varphi^{\prime}(\bar{y}) \wedge z=\omega \wedge \bar{x} \in I \wedge \bar{y} \in I \wedge z \in I\right)\right)$ is an upper bound of $(4, \exists \bar{x} \exists z(\varphi(\bar{x}) \wedge z=\omega \wedge \bar{x} \in I \wedge z \in I))$ and $\left(d, \varphi^{\prime}\right)$, and hence it is the least upper bound.

If $d=4 \omega$, then a minor variation of the above argument will work. Assume that $d=5$. In this case, equation (1) becomes

$$
\sup \left([(4, \exists \bar{x} \exists z(\varphi(\bar{x}) \wedge z=\omega \wedge \bar{x} \in I \wedge z \in I))]_{1},\left[\left(5, \varphi^{\prime}\right)\right]_{1}\right)=\left[\left(5, \varphi \wedge \varphi^{\prime}\right)\right]_{1},
$$

where $\varphi$ is $\exists \bar{x}(\varphi(\bar{x}) \wedge \bar{x} \in I)$. Since

$$
T^{*} \vdash \exists \bar{x} \exists z(\varphi(\bar{x}) \wedge z=\omega \wedge \bar{x} \in I \wedge z \in I) \rightarrow \exists \bar{x}(\varphi(\bar{x}) \wedge \bar{x} \in I)
$$

and

$$
T^{*} \vdash \varphi \wedge C^{5}(I) \rightarrow \exists \bar{x} \exists z(\varphi(\bar{x}) \wedge z=\omega \wedge \bar{x} \in I \wedge z \in I),
$$

it follows that equation (1) holds in this case also. 
Remark 27 Proposition 26 shows that simple relative degrees corresponding to $4 \omega$ can be eliminated from $S$ without loss of structure. In fact, this is very simple: one can express that $\omega \in I$ with a simple context, and this can be combined with degree 4. Although the introduction of the absolute degree $4 \omega$ is well motivated, and this degree represents a natural closure property, in the relative setting one can simulate the degree $4 \omega$ with the combination of degree 4 and simple contexts. Therefore, there is no reason to keep the distinction between the simple relative degrees $[(4 \omega, \exists \bar{x}(\varphi(\bar{x}) \wedge \bar{x} \in I))]_{1}$ and $[(4, \exists \bar{x} \exists z(\varphi(\bar{x}) \wedge z=\omega \wedge \bar{x} \in I \wedge z \in I))]_{1}$. The axiom of infinity is the only axiom with existence requirement $4 \omega$. It is not an accident that it is simply reducible: the nontrivial information of Theorem 20 is that it is simply reducible to degree 1 .

There is yet another natural preordering on the set $P$ of pairs $(d, \varphi)$, in which $d$ is an absolute degree and $\varphi$ is a context:

$$
(d, \varphi) \leq_{0}\left(d^{\prime}, \varphi^{\prime}\right) \quad \text { if and only if } \quad T^{*} \vdash \varphi^{\prime} \wedge C^{d^{\prime}}(I) \rightarrow \varphi \wedge C^{d}(I) .
$$

If $(d, \varphi) \leq_{0}\left(d^{\prime}, \varphi^{\prime}\right)$ and if the sentence $A$ admits degree $d$ of existence requirement relative to the context $\varphi$ in $T^{*}$, then $A$ admits degree $d^{\prime}$ of existence requirement relative to the context $\varphi^{\prime}$ in $T^{*}$. Moreover, $\leq_{1} \subset \leq_{0}$.

Once more, one can consider a canonical equivalence relation $\equiv_{0}$ associated to the preordering $\leq_{0}$, and the quotient of $P$ by $\equiv_{0}$. This quotient can be denoted by $Q_{0}$, and the partial ordering induced by $\leq_{0}$ on it can be denoted by the same symbol $\leq_{0}$. From $\leq_{1} \subset \leq_{0}$ it follows that $\equiv_{1} \subset \equiv_{0}$. Furthermore, if $(d, \varphi) \leq_{0}\left(d^{\prime}, \varphi^{\prime}\right)$ and $d \preceq d^{\prime}$, then $(d, \varphi) \leq_{1}\left(d^{\prime}, \varphi^{\prime}\right)$. On the other hand, if $(d, \varphi) \leq_{0}\left(d^{\prime}, \varphi^{\prime}\right)$ and $d^{\prime} \prec d$, then $(d, \varphi) \leq_{1}\left(d, \varphi^{\prime}\right)$ and $\left(d, \varphi^{\prime}\right) \equiv_{0}\left(d^{\prime}, \varphi^{\prime}\right)$.

Let $R$ denote the subset of $Q_{0}$ whose elements are the classes $[(d, \varphi)]_{0}$, in which $\varphi$ is a simple context, and let $H[S]$ denote the homomorphic image of $S$ under the homomorphism $H$ defined in Proposition 26.

Proposition 28 If $\varphi$ and $\varphi^{\prime}$ are simple contexts, then $(d, \varphi) \equiv_{0}\left(d^{\prime}, \varphi^{\prime}\right)$ if and only if $H\left([(d, \varphi)]_{1}\right)=H\left(\left[\left(d^{\prime}, \varphi^{\prime}\right)\right]_{1}\right)$. Moreover, $R$ is isomorphic to $H[S]$.

Proof Suppose that $H\left([(d, \varphi)]_{1}\right)=H\left(\left[\left(d^{\prime}, \varphi^{\prime}\right)\right]_{1}\right)$. If $d$ and $d^{\prime}$ are both different from $4 \omega$, then $[(d, \varphi)]_{1}=\left[\left(d^{\prime}, \varphi^{\prime}\right)\right]_{1}$. Since $\equiv_{1} \subset \equiv_{0}$, it follows that $[(d, \varphi)]_{0}=\left[\left(d^{\prime}, \varphi^{\prime}\right)\right]_{0}$. If $\max \left(d, d^{\prime}\right)=4 \omega$, then $H\left([(d, \varphi)]_{1}\right)=[(4, \psi)]_{1}$, $H\left(\left[\left(d^{\prime}, \varphi^{\prime}\right)\right]_{1}\right)=\left[\left(4, \psi^{\prime}\right)\right]_{1}$, and $[(4, \psi)]_{1}=\left[\left(4, \psi^{\prime}\right)\right]_{1}$, in which $\psi$ is the simple context equivalent to $\varphi \wedge \omega \in I$ and $\psi^{\prime}$ is $\varphi^{\prime} \wedge(\omega \in I)$. Therefore, $[(4, \psi)]_{0}=\left[\left(4, \psi^{\prime}\right)\right]_{0}$, and hence $[(4, \varphi)]_{0}=\left[\left(4, \varphi^{\prime}\right)\right]_{0}$.

Now, suppose that $(d, \varphi) \equiv_{0}\left(d^{\prime}, \varphi^{\prime}\right)$. If $d \neq d^{\prime}$, then one of $d, d^{\prime}$ is 4 and the other is $4 \omega$. In fact, if $(d, \varphi) \equiv_{0}\left(d^{\prime}, \varphi^{\prime}\right)$ and the sentence $A$ admits degree $d^{\prime}$ relative to $\varphi^{\prime}$, then $A$ admits degree $d$ relative to $\varphi$. If $A$ is an axiom of $Z F C$ and $r(A)=\max \left(d, d^{\prime}\right)$, then $r\left(A \mid \varphi \wedge \varphi^{\prime}\right)=\min \left(d, d^{\prime}\right)$, and hence $A$ is simply reducible. By Theorem 20, $\max \left(d, d^{\prime}\right)=4 \omega$. Suppose that $d^{\prime}=\max \left(d, d^{\prime}\right)=4 \omega$. By hypothesis, $T^{*} \vdash \varphi \wedge C^{d}(I) \rightarrow C^{4 \omega}(I)$, where $\varphi$ is a simple context; $d \prec 4$ would then cause the simple reducibility of the power set axiom. Again, by Theorem $20, d=4$.

For the second claim, consider the map $G: S \longrightarrow R,[(d, \varphi)]_{1} \mapsto[(d, \varphi)]_{0}$. Since $\equiv_{1} \subset \equiv_{0}$, the map $G$ is well defined. From the fact that $\leq_{1} \subset \leq_{0}$, it follows that $G$ is a homomorphism. By the first claim, the congruence associated with $G$ equals the congruence associated with $H$. Moreover, $G$ and $H$ are easily seen to be 
strong homomorphisms of partially ordered sets, in the sense defined in Mal'cev [3, p. 45]: if $H\left([(d, \varphi)]_{1}\right) \leq_{1} H\left(\left[\left(d^{\prime}, \varphi^{\prime}\right)\right]_{1}\right)$ and $d \preceq d^{\prime}$, then $[(d, \varphi)]_{1} \leq_{1}\left[\left(d^{\prime}, \varphi^{\prime}\right)\right]_{1}$. On the other hand, if $d^{\prime} \prec d$, then, as shown above, $d=4 \omega$ and $d^{\prime}=4$. In this case, $[(d, \varphi)]_{1} \leq_{1}\left[\left(d, \varphi^{\prime}\right)\right]_{1}$ and $H\left(\left[\left(d, \varphi^{\prime}\right)\right]_{1}\right)=H\left(\left[\left(d^{\prime}, \varphi^{\prime}\right)\right]_{1}\right)$, and $H$ is a strong homomorphism. The fact that $G$ is a strong homomorphism follows from the (already proved) fact ${ }^{4}$ that if $(d, \varphi) \leq_{0}\left(d^{\prime}, \varphi^{\prime}\right)$, then there is a pair $(e, \psi)$ such that $\psi$ is a simple context, $(d, \varphi) \leq_{1}(e, \psi)$, and $(e, \psi) \equiv_{0}\left(d^{\prime}, \varphi^{\prime}\right)$. The result follows from the homomorphism theorem in [3, pp. 45-46].

Remark 29 The introduction of $R$ explored the possibility of identification of pairs with different absolute degrees. Proposition 28 shows that the identification of $(4 \omega, \varphi)$ with $(4, \varphi \wedge(\omega \in I))$ is the only one achieved by $R$. The proof of this proposition shows that it is not possible to have a similar identification with other degrees and simple contexts. In fact, such an identification would cause the reducibility of an axiom that was proved to be simply irreducible. It follows that the semilattice $H[S]$ (or $R$ ) is very canonical for evaluating productivity: two natural paths from $P$ lead to $H[S]$.

\section{Final Remarks}

The main contribution of this work on the foundations of set theory is not in a particular result but rests in the conceptual apparatus developed for a systematic study of existence and related notions. This second part has focused on the notion of relative productivity, and the classification of the axioms of $Z F C$ in the semilattices $S$ and $R$ of simple relative degrees was achieved. Nevertheless, the apparatus can also be used to analyze the existential import of other principles independent of $Z F C$.

The notion of absolute productivity can be recovered in Definition 4 of productivity relative to a context: it suffices to use a tautological context. This formulation of absolute productivity is slightly different from the corresponding formulation in [1]. Although these formulations are only equivalent for definable domains $I$ (see Remark 6), the results are independent of the specific formulation. One can even employ stronger set theories with classes and give model-theoretic formulations for the notions of productivity and relative productivity. In this case, the proofs will no longer be finitary, but the same results will be accomplished.

One of the reasons for considering relative degrees was stated in the introduction: an enrichment of the structure of degrees of existence requirement can be used to evaluate the classification of the axioms obtained in [1]. However, that is by no means the only reason for considering relative degrees. In fact, the classification in the semilattice $S$, or in $R$, more accurately captures the productivity of a statement in general and is not intended to work for axioms only. This improvement in the evaluation of productivity already appears among the axioms: the classification of the axiom of infinity according to both $S$ and $R$ seems to be more correct than the classification in terms of absolute degrees.

There is a further motivation for the introduction of relative degrees, the evaluation of the productivity of combinatorial principles. One difficulty in evaluating the productivity of combinatorial principles is that the statement of these principles may already require a great deal of structure: it may require the existence of $\aleph_{1}$, of $\wp(\omega)$, and so forth. It hardly seems adequate to just rewrite these statements as equivalent sentences in $L(Z F)$ and evaluate these sentences in terms of absolute degrees. 
A more satisfactory approach would be achieved by taking the required structure for granted and evaluating how much productivity the principle adds over this structure. This can be done by restricting the domains in the evaluation of degrees to the ones that already contain the sets that must exist for the statement to be meaningful, that is, by evaluating the degree of existence requirement of the statement relative to an appropriate context.

Of course, not every context is coherent with the notion of productivity; every sentence admits degree 0 relative to some context. This justifies the focus on simple contexts. The simple contexts naturally lead to the simple relative degrees and to the semilattices $S$ and $R$. This is a great improvement on the finite linear order of absolute degrees. In fact, the semilattice $R$, which is isomorphic to $H[S]$, the homomorphic image of $S$ under the homomorphism $H$ of Proposition 26, is, arguably (see Remark 29), the correct structure for evaluating the productivity of sentences in set theory. However, the simple contexts are not the only relevant kind of context for relative productivity. Relative productivity can be used to compare the productivity of sentences in more than one way.

The results obtained in the present paper reinforce and extend the analysis in Part I. The main point of disagreement between the standard view and the analysis contained in this two-part work concerns the extensionality axiom. Extensionality asserts that if two sets are different, then there is an element in their symmetric difference. The validity of this assertion requires that, if two sets exist, then their elements also exist. Of course this is a very minor requirement, and transitivity is sufficient to fulfill this demand in a domain of existence. However, there is some requirement, and this demand is not fulfilled without transitivity. Furthermore, transitivity is not a first-order property, and one cannot just include this requirement in the axiom to solve the problem.

This second part has remained faithful to the semantic spirit of the first part. Only semantical definitions have stability under deduction and are not changed by syntactical variations. The understanding of the existence requirements of a sentence cannot come from its syntactical appearance alone. Rather, one should look at the semantical behavior of the sentence in domains of existence. This continues to hold in the relative setting.

\section{Notes}

1. The present article deals only with nonempty domains. The hypothesis $\exists x(x \in I)$ will be omitted, but one must keep in mind that, throughout this paper, $I$ is supposed to be nonempty.

2. For degree 0 these relations coincide: $(0, \varphi) \equiv_{1}\left(d^{\prime}, \varphi^{\prime}\right)$ if and only if $(0, \varphi) \equiv_{2}\left(d^{\prime}, \varphi^{\prime}\right)$.

3. The only exception is that the closure condition $C^{4}(I)$ can be raised to $C^{4 \omega}(I)$ by conjunction with a simple context (see Proposition 26).

4. This fact is proved shortly after the introduction of the relation $\leq_{0}$, in the next-to-last paragraph before the statement of Proposition 28. 


\section{References}

[1] Freire, R. A., "On existence in set theory," Notre Dame Journal of Formal Logic, vol. 53 (2012), pp. 525-47. MR 2995418. DOI 10.1215/00294527-1722737. 91, 92, 93, 94, 97, 99, 103

[2] Levy, A., Basic Set Theory, reprint of the 1979 original, Dover Publications, Minneola, N.Y., 2002. MR 1924429. 92, 93, 94, 97, 99

[3] Mal'cev, A., Algebraic Systems, posthumous edition, vol. 192 of Die Grundlehren der Mathematischen Wissenschaften, Springer, New York, 1973. MR 0349384. 103

\section{Acknowledgment}

Author's work supported by the State of São Paulo (Brazil) Research Foundation (FAPESP), process no. 2009/10751-8.

Centre for Logic, Epistemology and the History of Science

State University of Campinas

13083-859 Campinas, São Paulo

Brazil

freire@cle.unicamp.br 